Gynäkologische Endokrinologie 2010 • 8:263-270 DOI 10.1007/s10304-010-0384-8

Online publiziert: 3. Oktober 2010

(c) Springer-Verlag 2010

\section{Redaktion}

M.Birkhäuser, Basel

O. Ortmann, Regensburg

\section{Birkhäuser}

Prof. em. für gynäkologische Endokrinologie

und Reproduktionsmedizin der Universität Bern, Basel

\section{Risiko venöser und arterieller Thrombembolie}

\section{Ausgangslage}

Das thrombembolische Risiko unter kombinierten Ovulationshemmern $(\mathrm{OH})$ wurde in den letzten 30 Jahren durch die Entwicklung der Mikropillen $(\leq 35 \mu \mathrm{g}$ Ethinylestradiol, EE) signifikant gesenkt. Nach einigen, aber nicht allen Studien beeinflussen neben EE auch die Gestagene die Hämostase und spielen daher beim Thromboserisiko eine Rolle [3, 27, 28, 67, $71,72]$. Eine leicht vermehrte Resistenz auf aktiviertes Protein C, ein Anstieg von Prothrombin oder Faktor VII und ein Abfall des Faktors V bei Drittgenerationspillen und $\mathrm{OH}$ mit Drospirenon (gelegentlich als „vierte Generation“ bezeichnet) könnten zu einer höheren Koagulabilität führen [3, 67, 71]. Danach wären $\mathrm{OH}$ mit solchen Gestagenen im Vergleich zu OH der zweiten Generation, die meist Levonorgestrel enthalten, mit einem höheren Thromboserisiko assoziiert [28]. Reanalysen dieser Daten und neuere Untersuchungen zu den Zweit- und Drittgenerationspräparaten $[4,14,15,19,20,21$, $27,30,37,56,57,59]$ sind allerdings widersprüchlich. Bei den geringen Risikounterschieden könnte es sich um einen Bias durch eine für ältere und neuere $\mathrm{OH}$ unterschiedliche Verschreibungspraxis handeln, in dem neue $\mathrm{OH}$ als „Hoffnungsträger" v. a. bei Patientinnen mit Nebenwirkungen unter älteren $\mathrm{OH}$ und bei Erstanwenderinnen mit relativen Kontraindikationen eingesetzt wurden.

\title{
Hormonale Kontrazeptiva der zweiten, dritten und vierten Generation
}

\section{(7) Das Risiko für VTE ist immer entscheidend durch die Prädisposition der einzelnen Frau mitbestimmt}

Da OH mit dem Gestagen Drospirenon erst etwa 10 Jahre später als diejenigen mit den sog. Drittgenerationsgestagenen auf den Markt kamen, wurden sie in den älteren Diskussionen zum Risiko für venöse Thrombembolie (VTE) noch nicht einbezogen. Die seither publizierten Daten zu Drospirenon $[9,12,36,56,70]$ werfen für das VTE-Risiko dieser neueren Pillen wiederum ähnliche Fragen zur Ursache eines möglicherweise erhöhten VTE-Risikos auf $[8,20,29,30,33]$. Es muss aber bereits hier festgehalten werden, dass das Risiko für VTE immer entscheidend durch die Prädisposition der einzelnen Frau mitbestimmt ist.

\section{Epidemiologische Grundlagen}

Risiko bei gesunden, nichtschwangeren Frauen ohne OH-Einnahme

Ältere Arbeiten zur Inzidenz von VTE gingen in der gesunden Normalbevölkerung (nichtschwangere Frauen ohne $\mathrm{OH}$ ) von einer Inzidenz von 1 Fall auf 10.000 Frauenjahre aus. Durch die in den letzten Jahrzehnten eingetretenen demografischen Veränderungen (Anstieg des durchschnittlichen BMI, andere Lebensführung) und die Verbesserung der medizinischen Möglichkeiten (bessere Diagnostik und somit bessere Erfassung von VTE) wird heute die Inzidenz von VTE bei der gesunden Frau ohne $\mathrm{OH}$-Einnahme in der reproduktiven Lebensphase im Alter von 15-44 Jahren auf 3-5 pro 10.000 Frauenjahre geschätzt $[36,50,62,63,65,68]$. Dabei ist unbestritten, dass die Inzidenz von VTE altersabhängig ist $[4,29,30,36,51,55,61,62,68]$. Für gesunde, junge Frauen von weniger als 35 Jahren geht deshalb die Canadian Consensus Guideline on Continuous and Extended Hormonal Contraception von 2007 von einem niedrigeren absoluten Risiko für VTE von 1-2 Ereignissen auf 10.00o Frauenjahre aus [4].

Bei Frauen mit und ohne $\mathrm{OH}$ zu berücksichtigende nichthormonale Risikofaktoren für VTE beinhalten das Älterwerden [61], Antiphospholipidantikörper und hereditäre Thrombophilie [31, 49, 72], operative Eingriffe [16], Trauma und Immobilität, wozu längere Reisen und Hospitalisierung durch Krankheit oder Unfälle gehören [6, 41], Adipositas [35, 38, 49] und bei älteren Frauen möglicherweise Rauchen [47].

\section{Risiko in der Schwangerschaft und im Postpartum}

Die Inzidenz von VTE in der Schwangerschaft ist altersabhängig und gegenüber gesunden nichtschwangeren Frauen ohne $\mathrm{OH}$ um den Faktor 4-8 signifikant erhöht 
Tab. 1 Venöse und arterielle thrombembolische Risiken bei jungen Frauen

A) Risiko venöser thrombembolischer Ereignisse bei jungen Frauen

1. Bei Frauen unter 35 Jahren

Gesunde junge Frauen ohne $\mathrm{OH}$

1-2 Ereignisse/10.000 Frauenjahre (a)

Gesunde junge Schwangere am Termin

8-12 Ereignisse/10.000 Schwangerschaften (b)

Gesunde junge Frauen unmittelbar postpartal $200-400$ Ereignisse/10.000 Schwangerschaften (b)

Gesunde junge Frauen unter $\mathrm{OH}$

2-3 Ereignisse pro 10.000 Frauenjahre (c)

2. Bei Frauen im fertilen Alter (bis 44 Jahre) insgesamt

Gesunde junge Frauen ohne $\mathrm{OH}$

3-5 Ereignisse/10.000 Frauenjahre (a)

Gesunde junge Schwangere am Termin

8-40 Ereignisse/10.000 Schwangerschaften (b)

Gesunde junge Frauen unmittelbar postpartal

Gesunde junge Frauen unter $\mathrm{OH}$

Bis $>400$ Ereignisse/ 10.000 Schwangerschaften (b)

3. Mortalität an Lungenembolien bei jungen Frauen im fertilen Alter (d)

Die Mortalität wird bei VTE auf 1-2\% geschätzt

- Absolutes Risiko bei Nicht-Anwenderinnen

- Absolutes Risiko bei $\mathrm{OH}-A n w e n d e r i n n e n$

Ca. 5 Todesfälle auf 1 Mio. Frauen

B) Risiko von Herzinfarkten bei jungen Frauen (e)

Gesunde junge Frauen ohne $\mathrm{OH}$

Gesunde junge Schwangere

Gesunde junge Frauen unter $\mathrm{OH}$

Ca. 0,1 Ereignis/10.000 Frauenjahre

Ca. 0,62 Ereignisse/10.000 Schwangerschaften

Ca. 0,2 Ereignisse/10.000 Frauenjahre

(Raucherinnen: s. Text!)

C) Risiko von zerebrovaskulären Insulten bei jungen Frauen (f)

Gesunde junge Frauen ohne $\mathrm{OH}$

Ca. 1 Ereignis/10.000 Frauenjahre

Gesunde junge Schwangere

3-4 Ereignisse/10.000 Schwangerschaften

Gesunde junge Frauen unter $\mathrm{OH}$

Ca. 1 Ereignis/10.000 Frauenjahre

Literatur: a) $[4,36,50,62,63,65,68]$. b) $[4,22,24,26,33,40,48,50,51,53,68]$. c) $[2,4,9,13,19,23,29,36,50,55$,

61, 67]. d) $[4,52,62]$. e) $[1,4,25,39,42,45,46,53]$.f) $[1,4,5,18,39,46,53]$.

$[22,24,40,48,50,51,53]$. Sie liegt damit bei 8-40 Ereignissen pro 10.000 Schwangerschaften. Nach der Review von Heit et al. [22] beträgt das mittlere relative Risiko (standardisierte Inzidenz-Ratio) für VTE bei Schwangeren 4,29 (95\%-KI, 3,49-5,22; $\mathrm{p}<0,001$ ), das mittlere absolute Risiko (totale Inzidenz von VTE) 19,97 pro 10.00o Frauenjahre [22]. Die Inzidenz ist altersabhängig. Marik et al. [40] schließen in ihrer neueren Übersicht für jüngere Frauen auf eine etwas niedrigere absolute VTE-Inzidenz von 7,6-17,2 auf 10.000 Schwangerschaften. Die Inzidenz von Lungenembolien (LE) wird im Mittel mit 4,79 pro 10.00o Schwangerschaften angegeben. Marik et al. [40] zitieren für Europa und die USA eine Mortalität an LE von 1,1-1,5 pro 100.000 Geburten. Insgesamt ist im Postpartum im Mittel das Risiko 5-mal höher als bei Schwangeren (51,1 vs. 9,58 pro 10.000 Schwangerschaften), unmittelbar postpartal wird die absolute Inzidenz auf 200-400 VTE auf 10.000 Geburten geschätzt $[22,24,26,40$, $48,50,51,53]$. Auch das arterielle thrombembolische Risiko (Schlaganfälle und kardiale Ereignisse) steigt um das 3- bis 4-Fache an $[24,25]$. James et al. [24, 25, 26] schätzen für die USA die mittlere globale Prävalenz von venösen und arteriellen thrombembolischen Ereignissen in der Schwangerschaft auf ungefähr 20 pro $10.000 \mathrm{Ge}$ burten (ca. 80\% venös, $20 \%$ arteriell). VTE sind nach dieser Arbeit für 1,1 Todesfälle auf 100.000 Geburten verantwortlich, also für durchschnittlich 10\% [40]. In Großbritannien werden $30 \%[24,26,69]$ aller mütterlichen Todesfälle als Folge von VTE angenommen.

Die zum Vergleich der Mortalität unter $\mathrm{OH}$ und derjenigen während einer Schwangerschaft gelegentlich zitierte schwedische Publikation von Samuelsson et al. [54] unterscheidet sich von allen andern bekannten Daten, indem sie eine signifikant niedrigere Inzidenz von VTE in der Schwangerschaft angibt. Da diese Daten isoliert dastehen, kommen Samuelsson et al. [54] selbst zum Schluss, dass die benützten standardisierten nationalen Mortalitätsstatistiken bei jungen Frauen aus methodologischen Gründen kein genaues Monitoring der effektiven Mortalität an VTE erlauben. Somit darf die Arbeit von
Samuelsson et al. [54] nicht zum Vergleich mit dem VTE-Risiko unter $\mathrm{OH}$ beigezogen werden, wie dies gelegentlich getan wird.

Eine Zusammenstellung der neueren Literatur zum VTE-Risiko in Schwangerschaft und Postpartum bei Frauen unter 35 Jahren, das als Vergleichsbasis zum Risiko unter $\mathrm{OH}$ bei jüngeren Frauen beigezogen werden kann (• Tab. 1), findet sich in der Risikoanalyse der kanadischen Empfehlungen zur Kontrazeption von 2007 [4]. Für gesunde Schwangere unter 35 Jahren werden hier folgende absolute Risiken angegeben: am Termin 1012/10.00o Frauen, unmittelbar postpartal 200-400/10.00o Frauen [4].

\section{Risiko für venöse VTE unter Einnahme von Ovulationshemmern}

Die heute verschriebenen kombinierten Pillen mit einem EE-Gehalt von $<50 \mu$ g besitzen ein signifikant geringeres VTE-Risiko als solche mit $50 \mu \mathrm{g}$ EE oder mehr [17]. Nach den (stark variierenden) Zahlen aus neueren Metaanalysen und Kohortenstudien verdoppelt sich im Durchschnitt unter niedrig dosierten kombinierten $\mathrm{OH}$ die Inzidenz von VTE und liegt altersabhängig zwischen 2,1 und 10 Fällen pro 10.0oo Frauen jährlich $[9,13,19,21,30,36$, 50, 55, 56, 67, 70, 71]. Für gesunde junge Frauen unter 35 Jahren kommen die kanadischen Empfehlungen von 2007 [4] unter $\mathrm{OH}$-Einnahme auf ein VTE-Risiko von 23/10.0oo Frauenjahre. Diese Empfehlungen halten in Übereinstimmung mit der FDA fest, dass das absolute Risiko, unter $\mathrm{OH}$ ein thrombembolisches Ereignis (VTE) zu erleiden, klein ist [4]. Kanada unterscheidet wie die FDA nicht zwischen $\mathrm{OH}$ mit verschiedenen Gestagenen (s. unten).

\section{- Das VTE-Risiko ist v. a. bei}

Erstanwenderinnen und im ersten Anwendungsjahr erhöht.

Am höchsten ist es in den ersten 3 Monaten der $\mathrm{OH}$-Einnahme $[2,4,9,13,19,23$, 29, 36, 63], In der Regel demaskiert das initial erhöhte VTE-Risiko eine vermutlich vorbestehende Thrombophilie. Die prokoagulatorischen Effekte der $\mathrm{OH}$ benötigen 4 Wochen, um wieder in den Normalbereich abzusinken [4]. Daher besteht kein Grund zur Annahme, dass die 7-tä- 
gige Pillenpause einer zyklischen Pilleneinnahme einen günstigen Effekt auf das VTE-Risiko hat oder dass umgekehrt eine fix kombinierte $\mathrm{OH}$-Einnahme schädlich sein könnte. Nach Gomes et al. [19] sinkt das unter $\mathrm{OH}$ erhöhte Risiko innerhalb von 3 Monaten nach Absetzen wieder auf das altersentsprechende Basisrisiko ab.

Etwa 20\% der betroffenen Frauen entwickeln ein invalidisierendes postthrombotisches Syndrom, und etwa 10\% erleiden eine Lungenembolie. Die Mortalität wird bei VTE auf 1-2\% geschätzt [52, 61, 62] und liegt somit zwischen 15 und 20 Todesfällen pro 1 Mio. Anwenderinnen pro Jahr. Zum Vergleich wird davon ausgegangen, dass unter Nichtanwenderinnen von $\mathrm{OH}$ ca. 5 Frauen auf 1 Mio. pro Jahr an einer LE versterben.

Theoretisch sollte eine weitere Dosisreduktion von EE das VTE-Risiko zusätzlich senken [1]. Allerdings konnte diese Annahme bisher trotz mehrerer Hinweise aus neueren Studien nicht eindeutig gesichert werden $[9,36]$.

Die nichtorale Verabreichung (Vaginalringe, Pflaster) einer Kombination von EE mit einem Gestagen senkt das Risiko nach den zur Verfügung stehenden Daten nicht [10].

Das Risiko für VTE unter $\mathrm{OH}$ wird entscheidend durch folgende individuelle Risikofaktoren erhöht:

- Älterwerden [36, 44, 61],

- Vorhandensein von Antiphospholipidantikörpern und einer hereditären Thrombophilie $[2,3,31,49,67,71,72]$,

- Adipositas [35, 38, 44, 49],

- operative Eingriffe [16],

- Trauma, Unfälle und Immobilisierung $[6,44,64]$ sowie

- Flugreisen $[6,41]$.

\section{Risiko für arterielle thrombotische Erkrankungen unter Einnahme von Ovulationshemmern}

\section{Herzinfarkt}

Unter $\mathrm{OH}$ könnte das Risiko für Herzinfarkte altersabhängig bis um das 4 -Fache erhöht sein $[1,28,32,45,46,66]$, doch betrifft dieser Risikoanstieg anscheinend ausschließlich Raucherinnen und Frauen mit arterieller Hypertonie. Zum Vergleich ist bei Schwangeren das Herzinfarktrisiko höher als bei Nichtraucherinnen unter

Gynäkologische Endokrinologie 2010 · 8:263-270 DOI 10.1007/s10304-010-0384-8

(c) Springer-Verlag 2010

\section{Birkhäuser \\ Hormonale Kontrazeptiva der zweiten, dritten und vierten Generation. Risiko venöser und arterieller Thrombembolie}

\section{Zusammenfassung}

Für die Verschreibung von Ovulationshemmern $(\mathrm{OH})$ sind nicht nur die venösen thrombembolischen Ereignisse entscheidend, sondern die Summe aller Nebenwirkungen. Für die Praxis ergeben sich aus der heutigen Datenlage folgende Konsequenzen: 1. Venöse Thrombembolien sind Teil der unerwünschten, jedoch seltenen Nebenwirkungen kombinierter hormonaler Kontrazeptiva. Das Risiko einer Thrombose ist im ersten Anwendungsjahr und insbesondere in den ersten 3 Monaten am höchsten. 2. Ein unterschiedliches Risiko für venöse Thrombembolie (VTE) unter älteren $\mathrm{OH}$ einerseits und neueren Pillen andererseits ist bei gesunden vergleichbaren Populationen nicht gesichert. Entscheidend für das Risiko sind die Prädisposition, das Alter, persönliche Risikofaktoren sowie die Respektierung der klassischen Kontraindikationen. 3. Herzinfarkte und CVI sind bei jungen Frauen so selten, dass die verfügbaren Studien keine definitiven Schlussfol- gerungen erlauben. Bei Nichtraucherinnen scheint das Risiko nicht erhöht zu sein. 4. Bei jungen gesunden Frauen ohne erkennbare Risikofaktoren müssen bei Erstverschreibungen Zweitgenerationspräparate gegenüber neueren $\mathrm{OH}$ nicht bevorzugt werden. 5 . Das VTE-Risiko ist unter nichtoralen kombinierten hormonalen Kontrazeptiva nicht geringer als unter einer kombinierten oralen hormonalen Kontrazeption. 6. Bei Vorliegen von Risikofaktoren und bei Verdacht auf eine Thrombophilie sollen reine Gestagenpräparate, ein Gestagen freisetzendes IUD oder eine nichthormonelle Methode eingesetzt werden.

\section{Schlüsselwörter}

Hormonale Kontrazeptiva .

Ovulationshemmer $\cdot$ Venöse

Thrombembolie · Thrombophilie .

Thrombose · Gestagene

\section{Hormonal contraceptives of the second, third, and fourth generation. Risk of venous and arterial thromboembolism}

\section{Abstract}

Not only venous thromboembolic events but also the sum of all side effects are decisive for the prescription of oral contraceptives. Based on the data available today, the following conclusions may be drawn for the clinical management: 1 . Venous thromboembolisms are among the undesirable, but rare side effects of hormonal contraceptives. The risk of thrombosis is highest in the first year of use, particularly during the first 3 months. 2. There is no evidence for a different risk of venous thromboembolism between older oral contraceptives and newer pills in comparably healthy populations. The crucial aspects determining risk are predisposition, age, personal risk factors, and heeding the classic contraindications. 3. Myocardial infarction and chronic venous insufficiency are so uncommon in young women that available studies do not permit definitive conclu- sions to be drawn. In nonsmokers the risk does not appear to be elevated. 4. In healthy young women without recognizable risk factors preference must be given to second-generation drugs over newer oral contraceptives for first-time prescriptions. 5. The risk of venous thromboembolism is not lower under non-oral combined hormonal contraceptives than under combined oral hormonal contraception. 6 . In the presence of risk factors and when thrombophilia is suspected, progestinonly preparations, a progestin-releasing IUD, or a non-hormonal method should be employed.

\section{Keywords}

Contraceptives, hormonal · Contraceptives, oral - Venous thromboembolism . Thrombophilia · Thrombosis · Progestagens 
$\mathrm{OH}$ [25]. Mant et al. [39] beobachteten ein erhöhtes Herzinfarktrisiko ausschließlich bei Frauen, die bereits bei Studienbeginn schwere Raucherinnen waren. Unter $\mathrm{OH}$ fand sich ein zusätzlicher Herzinfarkt auf 106o starke Raucherinnen [39]. Deshalb sollte der Zigarettenkonsum vor dem Beginn einer OH-Einnahme gestoppt und $\mathrm{OH}$ an Raucherinnen über 35 Jahre prinzipiell nicht abgegeben werden. Die Women's Ischemia Syndrome Evaluation (WISE) Study [42] stellte fest, dass postmenopausale Frauen, die früher $\mathrm{OH}$ eingenommen hatten, bei der quantitativen angiographischen Beurteilung einen niedrigeren Score für koronare Herzkrankheit besitzen als Frauen, die nie $\mathrm{OH}$ benützt hatten.

\section{(>) OH sollten an Raucherinnen über 35 Jahre prinzipiell nicht abgegeben werden}

Es gibt Hinweise dafür, dass das Risiko für Herzinfarkte unter $\mathrm{OH}$ der dritten Generation und solchen mit Drospirenon geringer ist als unter Zweitgenerationspillen [9, 34]. Die gefundenen Risikounterschiede sind aber nicht signifikant.

\section{Zerebrovaskuläre Insulte}

Es ist umstritten, ob außer bei starken Raucherinnen unter modernen, niedrig dosierten $\mathrm{OH}$ überhaupt ein erhöhtes $\mathrm{Ri}$ siko für zerebrovaskuläre Insulte (CVI) besteht $[5,9,18,25,32,34,39,60,66]$. In einer Studie [39] wurde über einen 3-fachen Risikoanstieg für CVI berichtet, doch ist das absolute Basisrisiko in dieser Altersgruppe sehr gering: Absolut handelte es sich um einen zusätzliche Fall eines CVI auf 5880 Pillenanwenderinnen. Bei gesunden jungen Frauen mit normalem arteriellem Blutdruck wurde in einer neueren Studie kein Zusammenhang zwischen $\mathrm{OH}$ und CVI gefunden [42]. Auch wenn das absolute Risiko aller kardio- und zerebrovaskulären Erkrankungen unter $\mathrm{OH}$ mit demjenigen während einer Schwangerschaft verglichen wird [25], bleibt es sehr klein. Ein solcher Vergleich scheint legitim, da bei unzuverlässiger Kontrazeption immer das Risiko einer ungeplanten Schwangerschaft besteht.

\section{Risiko venöser thrombotischer Ereignisse bei neueren Ovulationshemmern mit Drospirenon und Cyproteron-Acetat}

Zwei $2007[9,56]$ und eine 2010 [7] publizierte prospektive Beobachtungsstudien zeigen für $\mathrm{OH}$ mit Drospirenon, mit Cyproteron-Acetat und für sog. Drittgenerationspräparate im Vergleich zu älteren $\mathrm{OH}$ der sog. zweiten Generation kein erhöhtes Risiko. Für $\mathrm{OH}$ mit CyproteronAcetat [9] und Drospirenon [9, 56] liegt das VTE-Risiko im Bereiche der Zweitgenerationspillen. Beide Studien wurden von der Herstellerfirma durch einen „unrestricted grant" unterstützt.

Die European Active Surveillance Study („EURAS“) wurde in Praxen und Familienplanungszentren von 7 europäischen Ländern (Belgien, Dänemark, Deutschland, Frankreich, Großbritannien, Holland, Österreich) durchgeführt [9]. Sie erfasste insgesamt 142.475 Frauenjahre bei 59.674 Studienteilnehmerinnen. Im Verlaufe der Studie wechselten $20,8 \%$ (Drospirenon-Kohorte), 22,3\% (Levonorgestrel-Kohorte) respektive $26,9 \%$ (andere Pillen) der Studienteilnehmerinnen von ihrem initialen Präparat auf eine andere Pille oder eine nichtorale hormonale Methode. Die Drop-outRate betrug 2,4\%. Alle Diagnosen wurden aufgrund einer Durchsicht der Krankengeschichten validiert und insgesamt 118 VTE ermittelt. Für alle Kohorten mit $\mathrm{OH}$ und $\mathrm{NOH}$ (nichtorale hormonale Kontrazeption) wurde eine ähnliche VTE-Inzidenz gefunden. Die VTE-Inzidenz betrug für die Drospirenon-Kohorte 26 Fälle respektive 9,1 Ereignisse pro 10.000 Frauenjahre, für Levonorgestrel 25 Fälle respektive 8,o Ereignisse pro 10.000 Frauenjahre, für andere $\mathrm{OH} 52$ VTE-Fälle respektive 9,9 Ereignisse pro 10.000 Frauenjahre und für die deutlich kleinere NOH-Kohorte 3 Fälle respektive 7,4 Ereignisse pro 10.00o Frauenjahre. Im Vergleich zu Drospirenon betrug die korrigierte Hazard Ratio für VTE für Levonorgestrel 1,0 (95\%-Vertrauensintervall $0,6-1,8)$ und für andere $\mathrm{OH}$ o,8 (o,5-1,3; [9]). Somit fand sich in der EURAS-Studie [9] hinsichtlich der VTE-Raten kein Unterschied zwischen den Drospirenon-haltigen neueren Pillen und den anderen verschriebenen Präparaten, obwohl Drospirenon- haltige $\mathrm{OH}$ häufiger bei Frauen mit einem höherem BMI verschrieben wurden [9].

Die zweite prospektive Studie (INGENIX claims database; $[12,56])$ wurde in den USA bei Frauen im Alter von 10-59 Jahren durchgeführt. Die Beobachtungsdauer betrug im Mittel 7,6 Monate. Zur Auswertung wurden nach einem speziellen prädiktiven statistischen Modell („propensity score") 22.429 Neuanwenderinnen eines $\mathrm{OH}$ mit Ethinylestradiol/Drospirenon mit 44.858 Neuanwenderinnen eines anderen $\mathrm{OH}$ im Verhältnis 1:2 in Kohorten gepaart. Um die Neuanwenderinnen verschiedener Pillen in Funktion des Gestagens (Drospirenon-haltige vs. andere Pillen) entsprechend ihrem Ausgangsrisiko für VTE zu matchen, benutzte diese Studie ein Scoring-System für Risikofaktoren. Die Richtigkeit der Diagnose „VTE“ wurde anhand der Krankengeschichten validiert. Die Datenanalyse ergab 18 Fälle von VTE bei Neuanwenderinnen von $\mathrm{OH}$ mit Drospirenon und 39 Fälle von VTE bei Neuanwenderinnen von $\mathrm{OH}$ mit anderen Gestagenen (Rate Ratio 0,9; 95\%Vertrauensintervall o,5-1,6). Zwischen der VTE-Inzidenz unter Drospirenon-haltigen $\mathrm{OH}$ und derjenigen unter anderen Pillen wurden somit auch in dieser Studie $[12,56]$ keine Unterschiede gefunden.

Eine 2010 publizierte deutsche prospektive Fall-Kontroll-Studie [7] bei 68o Fällen von VTE und 2720 dazu gepaarten Kontrollen stützt die Daten von 2007 [9, 12, 56], indem sie für $\mathrm{OH}$ mit den Gestagenen Drospirenon und Dienogest im Vergleich zu niedrig dosierten $\mathrm{OH}$ mit Levonorgestrel kein erhöhtes Risiko fand (korrigierte Odds Ratio Drospirenon- und Dienogest- vs. Levonorgestrel-haltige $\mathrm{OH}:$ 1,1, 95\%-Vertrauensintervall o,6-1,8). Leider unterscheidet diese Studie nicht zwischen OH mit Dienogest und solchen mit Drospirenon.

Im Jahr 2009 erschienen eine FallKontroll- und eine Kohortenstudie zum Thromboserisiko unter $\mathrm{OH}$, die beide $\mathrm{Da}$ ten zu Präparaten mit Drospirenon und Cyproteron-Acetat enthalten $[36,70]$. Deren Ergebnisse lassen eine leichte Erhöhung des Thromboserisikos (relatives Risiko 1,52) unter $\mathrm{OH}$ mit Gestagenen der dritten Generation und solchen mit Drospirenon oder Cyproteron-Acetat gegenüber Pillen der zweiten Generation vermuten.

Bei der holländischen Publikation von van Hylckama Vlieg et al. [7o] handelt es 
Hier steht eine Anzeige.

黛 Springer 
sich um eine retrospektive Post-hoc-FallKontroll-Studie innerhalb der holländischen MEGA-Studie. Die MEGA-Studie war ursprünglich darauf angelegt worden, den Einfluss von umweltbedingten und genetischen Faktoren auf VTE zu erfassen. Die zusätzliche retrospektive Post-hocFall-Kontroll-Studie wurde bei 1524 Patientinnen und 1760 Kontrollen durchgeführt, um die Wirkung verschiedener $\mathrm{OH}$ auf das Risiko für VTE zu erfassen. Als „Fälle“ wurden Frauen identifiziert, die wegen einer VTE in einer Antikoagulationsklinik behandelt wurden. Die Mehrzahl der dazu verwendeten Kontrollen waren Partnerinnen von Männern, die in derselben Klinik behandelt worden sind, andere Kontrollen wurden via Zufallskontakte telefonisch rekrutiert. Beide Rekrutierungsverfahren sind für Fall-Kontroll-Studien unüblich. Auch war nicht für alle Studienteilnehmerinnen bekannt, ob sie Neuanwenderinnen waren. Zudem wurde der Anstieg des Basisrisikos in der gesunden Normalbevölkerung im Verlaufe der letzten 20 Jahren und beim Matchen von Fällen und Kontrollen der BMI nicht berücksichtigt. Diese Faktoren beeinflussen das VTE-Risiko. Verglichen mit Nichtanwenderinnen betrug die ermittelte Odds Ratio [70] für Gestoden 5,6 (Vertrauensintervall 3,7-8,4), für Desogestrel 7,3 $(5,3-10,0)$, für Cyproteron-Acetat 6,8 $(4,7-10,0)$ und für Drospirenon $6,3(2,9-13,7)$. Somit überlappen sich in der holländischen MEGA-Studie die 95\%Vertrauensintervalle zu den Odd Ratios für alle untersuchten $\mathrm{OH}$ mit verschiedenen Gestagenen, sodass keine statistisch signifikanten Unterschiede im VTE-Risiko zwischen Drospirenon-haltigen und anderen Pillen erwartet werden dürften. Dennoch folgern die Autoren, dass Zweitgenerationspillen hinsichtlich des VTE-Risikos sicherer sind [70]. Die FDA kam in ihrer Beurteilung zum Schluss, dass die Anzahl von Fällen unter Drospirenon-haltigen Pillen in der MEGA-Studie mit 1,2\% aller beobachteten Fälle zu niedrig war, um eine zuverlässige Beurteilung des VTE-Risikos zu erlauben (www.fda.gov/Safety/MedWatch/ SafetyInformation/ucm211762.htm).

Die zweite von Lidegaard et al. [36] publizierte Arbeit, die über eine erhöhte VTE-Rate unter Drittgenerationspillen und solchen mit Drospirenon oder Cyproteron-Acetat berichtete, ist eine retrospek- tive dänische Kohortenstudie bei Frauen zwischen 15 und 49 Jahren, die sich auf das Dänische nationale Patienten-Register abstützt (s. unten). Im Beobachtungszeitraum (01.01.1995-31.12.2005) erfasste sie 3,4 Mio. Frauenjahre unter Pilleneinnahme, 2,3 Mio. Frauenjahre bei früherer Pilleneinnahme und 4,8 Mio. Frauenjahre bei Nichtanwenderinnen. Insgesamt wurden in der untersuchten Periode vom 01.01.1995 bis zum 31.12.2005 10,4 Millionen Frauenjahre erfasst [36]. Es fanden sich total 4213 erstmalige thrombembolische Ereignisse, 2045 davon unter Anwendung einer Pille [36]. Die VTE-Inzidenz stieg unter $\mathrm{OH}$ mit zunehmendem Alter an (von 1,84 pro 10.000 Frauenjahre im Alter von 15-19 auf 6,59 pro 10.000 Frauenjahre mit 45-49 Jahre). Auch sank in Übereinstimmung mit älteren Berichten das VTE-Risiko mit zunehmender Einnahmedauer (<1 Jahr 4,17, 95\%-Vertrauensintervall 3,734,66, 1-4 Jahre 2,98, 2,73-3,26, und >4 Jahre 2,76, 2,53-3,02; $\mathrm{p}<0,001) \mathrm{ab}$. Bei den Nichtanwenderinnen von $\mathrm{OH}$ betrug die mittlere Inzidenz von VTE zwischen 15 und 49 Jahren 3,01 Ereignisse pro 10.00o Frauenjahre. Verglichen zu OH mit Levonorgestrel und gleicher Ethinylestradioldosierung und Anwendungsdauer fand sich für alle Altersgruppen zusammengenommen (15-49 Jahre) eine Ratio, die für $\mathrm{OH}$ mit Norethisteron $0,98(0,71-1,37)$, mit Norgestimat 1,19 (0,96-1,47), mit Desogestrel 1,82 (1,49-2,22), mit Gestoden 1,86 (1,59$2,18)$, mit Drospirenon 1,64 (1,27-2,10) und mit Cyproteron-Acetat 1,88 $(1,47-2,42)$ betrug. Die Studie von Lidegaard et al. [36] kam zum Schluss, dass Drittgenerationspillen sowie solche mit Drospirenon und Cyproteron-Acetat im Vergleich zu Pillen mit Levonorgestrel ein leicht höheres VTERisiko besitzen. Dabei könnten allerdings wie bei der Diskussion Zweit-/Drittgenerationspillen wiederum methodische Probleme eine Rolle spielen wie die inkomplette Erfassung des bekannt höheren Risikos im ersten Anwendungsjahr bei den alten Präparaten mit einem Einnahmebeginn vor $1995[8,35,59]$ und die Nichtberücksichtigung der Zunahme des mittleren BMI in Dänemark seit 1995 [35]. Auch kann ein Selektionsbias vermutet werden, indem mehr Risikopatientinnen Präparate mit Drospirenon verschrieben bekamen als ältere $\mathrm{OH}$, wie dies zum Teil der Mar- ketingstrategie bei der Produkteinführung entsprach. In einem in diesem Jahr publizierten Artikel wiesen Severinsen et al. [58] in einem von der Lidegaard-Gruppe [36] unabhängigen Artikel darauf hin, dass die Austrittsdiagnose „VTE“ des Dänischen nationalen Patienten-Registers für die medizinische Forschung nur mit Vorsicht benutzt werden darf. Der positive prädiktive Wert, der auf den stationären Spitalabteilungen kodierten Diagnosen „VTE“ betrug 75,0\% (95\%-Vertrauensintervall: 71,9, 77,9), die von den Notfallstationen kodierten Diagnosen erwiesen sich als nicht verwertbar. Die Daten von Lidegaard et al. [36] werden daher zurzeit reanalysiert. Die Resultate dürften Ende 2010 vorliegen.

Insgesamt bleibt jedoch bei beiden Studien, der holländischen [70] und der dänischen [36] Arbeit, der Anstieg an VTE pro 10.000 Frauenjahre im Vergleich zu Frauen ohne $\mathrm{OH}$ in der gleichen Größenordnung wie bei den älteren Präparaten.

\section{Reaktion der Aufsichtsbehörden}

Wie haben die verschiedenen Aufsichtsbehörden reagiert? Die Swissmedic hält in ihrem Schlussbericht zum Risiko von VTE unter Drospirenon-haltigen $\mathrm{OH}$ fest [65]: „Für Drospirenon-haltige Pillen lag das relative Risiko in 2 großen, 2009 publizierten retrospektiven epidemiologischen Studien, einer dänischen Kohortenstudie und einer holländischen Fall-Kontrollstudie, zwischen jenem der 2. und 3. GenerationsKontrazeptiva und war gegenüber jenen der 2. Generation um einen Faktor 1.5 erhöht; die Risikoerhöhung in der holländischen Studie war jedoch statistisch nicht signifikant. Im Gegensatz dazu war dieses Risiko in zwei großen, prospektiven Kohortenstudien (EURAS und Ingenix) vergleichbar mit jenem der Kontrazeptiva der 2. Generation. (http://www.swissmedic. $\mathrm{ch} /$ )“ Die FDA folgerte (www.fda.gov/ Safety/MedWatch/SafetyInformation/ ucm211762.htm), dass die Anzahl von Fällen unter Drospirenon-haltigen Pillen in der MEGA-Studie mit 1,2\% aller beobachteten Fälle zu niedrig war, um eine zuverlässige Beurteilung des Risikos zu erlauben. Dagegen hielt die EMEA im PhVWP Monthly Report Nr 1003 fest [11]: „Recently, two epidemiological studies were published, both assessing the risk of VTE in cur- 
rent users of different types of COCs. The results suggested that the risk for the COCs containing $30 \mu \mathrm{g}$ of EE in combination with drospirenone might be higher than previously estimated, and between those of second and third generation pills." Die BfArM richtet sich nach diesen europäischen Empfehlungen (www.bfarm.de/cln 103/sid_F59FADE1D0357D6A2F19B5 DCB499B742/DE/Pharmakovigilanz/risikoinfo/yasmin.html).

\section{(2) Essenziell ist es, in der Familien- und Eigenanamnese nach Risikofaktoren zu fragen}

Die nach den Stellungnahmen der verschiedenen Heilmittelaufsichtsbehörden im Mai 2010 publizierte Übersichtsarbeit von Sehovic und Smith [57] kommt zum Schluss, dass die Ratio der Inzidenz für VTE bei Frauen unter OH mit Drospirenon im Vergleich zu OH mit anderen Gestagenen im Bereich von 0,9-1,7 (95\%-KI $0,5-2,4)$ liegt. Essenziell ist es, in der Familien- und Eigenanamnese nach Risikofaktoren, wie z. B. frischer Thrombembolie, Status nach tiefer Venenthrombose oder thrombembolischen Ereignissen, bekannter oder vermuteter Thrombophilie, Status nach Herzinfarkt oder nach zerebrovaskulärem Ereignis, bekannter kardiovaskulärer Erkrankung, arterieller Hypertonie, Migräne (v. a. mit Aura), Nikotinabusus, Diabetes mellitus (v. a. mit Angiopathien) oder Hyperlipidämie zu fragen, um nur Faktoren mit einem erhöhtem Risiko für venöse oder arterielle VTE zu erwähnen.

\section{Konsequenzen für die Praxis}

Aus dem Gesagten ergeben sich für die Praxis folgende Konsequenzen:

1. Venöse Thrombembolien sind Teil der unerwünschten, jedoch seltenen $\mathrm{Ne}$ benwirkungen kombinierter hormonaler Kontrazeptiva (• Tab. 1).

2. Ein unterschiedliches Risiko für VTE unter älteren $\mathrm{OH}$ einerseits und neueren Pillen andererseits ist bei vergleichbaren gesunden Populationen nicht gesichert. Entscheidend für das Risiko sind die Prädisposition, das Alter und persönliche Risikofaktoren (- Infobox 1). Zur Senkung des Risikos von VTE müssen die klassischen
Infobox 1 Hinweise auf erhöhtes Risiko für venöse und arterielle thrombembo-

lische Erkrankungen

1. Anamnestische Risikofaktoren

- Fortgeschritteneres Alter

- Rauchen

- Positive Familienanamnese für venöse oder arterielle Thrombembolien bei einem Geschwister oder einem Elternteil

- Adipositas (BMI >30 kg/m²)

- Störungen des Fettstoffwechsels (Dyslipoproteinämie)

- Hypertonie

- Migräne mit Aura

- Herzklappenerkrankungen

- Vorhofflimmern

- Längerfristige Immobilisierung, größere chirurgische Eingriffe, schwere Verletzungen, Eingriff/Frakturen untere Extremität

2. Erkrankungen mit ungünstigen Zirkulationsverhältnissen

- Diabetes mellitus

- Systemischer Lupus erythematodes (SLE)

- Hämolytisch-urämisches Syndrom

- Chronisch entzündliche Darmerkrankungen (M. Crohn, Colitis ulcerosa)

- Sichelzellenanämie

- Migräne

3. Gerinnungsphysiologische Gesamtabklärung bei Risikofaktoren

- Bei Vorliegen von Risikofaktoren sollte eine Thrombophilieabklärung durch ein Zentrum für Hämotologie/Gerinnungsphysiologie erfolgen, in der Regel mit konsiliarischer Überweisung an einen Gerinnungsspezialisten

- Bei negativem Ausfall des ThrombophilieScreenings und weiter bestehendem anamnestischem oder klinischem Verdacht auf Thrombophilie ist eine erhöhtes thrombembolisches Risiko nicht ausgeschlossen

Kontraindikationen respektiert werden (• Infobox 2).

3. Arterielle Thrombembolien, Herzinfarkte und CVI sind bei jungen Frauen so selten, dass die verfügbaren Studien aufgrund der geringen statistischen Signifikanz keine definitiven Schlussfolgerungen erlauben (• Tab. 1).

4. Das Risiko einer Thrombose unter $\mathrm{OH}$ ist im ersten Anwendungsjahr und insbesondere in den ersten 3 Monaten am höchsten. Dies gilt für alle $\mathrm{OH}$ der sog. zweiten, dritten und vierten Generation.

5. Da ein unterschiedliches Thromboserisiko zwischen den verschie-
Infobox 2 Gründe für das sofortige Absetzen von kombinierten hormonellen Kontrazeptiva

- Erstmaliges Auftreten oder Exazerbation von Migräne; häufigeres Auftreten ungewohnt starker Kopfschmerzen

- Plötzliche Seh-, Hör-, Sprech- oder sonstige Wahrnehmungsstörungen

- Erste Anzeichen von thrombembolischen Erscheinungen, insbesondere Atemnot, unklare Thoraxschmerzen oder Husten unklarer Ursache

- Unklare Schmerzen in einer Extremität und/oder Schwellung eines Beines, v. a. nach Flug- und Busreisen

- Mindestens 4 Wochen vor geplanten Operationen, während Immobilisierung (z. B. nach Unfall oder Operation); bei Frakturen/Operationen der unteren Extremität. Wo Absetzen nicht möglich, gezielte Thromboseprophylaxe!

- Signifikanter Blutdruckanstieg (bei wiederholter Messung)

- Verdacht auf Herzinfarkt oder koronare Herzkrankheit

- Verdacht auf zerebrovaskuläres Ereignis, TIA

- Auftreten von Ikterus, Hepatitis, generalisiertem Pruritus

- Starke Oberbauchschmerzen oder Lebervergrößerung

- Schwangerschaft oder Verdacht auf Schwangerschaft

denen Mikropillen aufgrund der widersprüchlichen Datenlage nicht gesichert ist, besteht kein Anlass, deshalb für Erstverschreibungen bei jungen gesunden Frauen ohne erkennbare Risikofaktoren Zweitgenerationspräparate gegenüber den neueren $\mathrm{OH}$ zu bevorzugen. Die Erstverschreibung von $\mathrm{OH}$ mit Drospirenon oder Cyproteron-Acetat ist insbesondere bei $\mathrm{Zu}$ satzindikationen wie Akne, Hirsutismus oder Alopezie weiterhin zu vertreten.

6. Bei gesunden Frauen, die bereits nebenwirkungsfrei eine Drittgenerationspille oder eine Pille mit Drospirenon respektive Cyproteron-Acetat verwenden und sich damit wohlfühlen, muss nicht auf ein anderes Präparat gewechselt werden.

7. Das VTE-Risiko ist unter nichtoralen kombinierten hormonalen Kontrazeptiva (Pflaster, Vaginalringe) nicht geringer als unter einer kombinierten oralen hormonalen Kontrazeption. 
8. Die Östrogendosis einer Pille ist innerhalb des Spektrums der Mikropillen hinsichtlich des VTE-Risikos für die Ersteinstellung bei gesunden jungen Frauen nicht allein ausschlaggebend. Insbesondere bei Adoleszentinnen und jungen oligoamenorrhöischen Frauen bleibt offen, welche Östrogendosis noch eine optimale Entwicklung der „peak bone mass" garantiert.

9. Vor jeder Verschreibung von $\mathrm{OH}$ muss in der Familien- und Eigenanamnese aktiv nach Risikofaktoren für VTE gefragt werden. Diese müssen regelmäßig reevaluiert werden. Jede Pillenanwenderin muss darüber informiert sein, wann sie einen $\mathrm{OH}$ absetzen muss (• Infobox 2).

10. Bei Vorliegen von VTE-Risikofaktoren und bei Verdacht auf eine Thrombophilie sind reine orale Gestagenpräparate, ein Gestagen abgebender IUD oder eine nichthormonelle Methode vorzuziehen.

\section{Fazit}

Für die Verschreibung von $\mathrm{OH}$ sind nicht nur die venösen thrombembolischen Ereignisse entscheidend, sondern die Summe aller, zum Teil auch günstiger Nebenwirkungen auf verschiedene Organe wie Knochen, Ovar oder Endometrium. Dazu gehören auch die metabolischen Folgen von $\mathrm{OH}$ sowie deren Wirkung auf die Blutungsstärke, ein prämenstruelles Syndrom und das allgemeine Wohlbefinden. Dabei muss jeder Patientin immer bewusst sein, dass ein $\mathrm{OH}$ ein Medikament mit möglichen Nebenwirkungen und kein Lifestyle-Präparat ist. Sofern angesichts der widersprüchlichen Literatur $[1,4,8,13,14,15,17,19,20,27,29,30,33$, $44,50,56,57,59,63,64,71]$ eine Differenz zwischen den verschiedenen Gestagenen bestehen sollte, so ist sie klein (1-2 zusätzliche Fälle/10.000 Frauenjahre [29,57]).

\section{Korrespondenzadresse \\ Prof. Dr. M. Birkhäuser}

Prof. em. für gynäkologische Endokrinologie und Reproduktionsmedizin der Universität Bern Gartenstr. 67, 4052 Basel

Schweiz

martin.birkhaeuser@balcab.ch

\section{Literatur}

1. Baillargeon JP, McClish DK, Essah PA, Nestler JE (2005) Association between the current use of lowdose oral contraceptives and cardiovascular arterial disease: a meta-analysis. J Clin Endocrinol Metab 90:3863-3870

4. Guilbert E, Richard BR (Hrsg) (2007) Candadian Consensus Guideline on Continuous and Extended Hormonal Contraception. J Obstet Gynaecol Can 29(Suppl 2/supplément 2):S1-S32

5. Chan WS, Ray J, Wai EK et al (2004) Risk of stroke in women exposed to low-dose oral contraceptives: a critical evaluation of the evidence. Arch Intern Med 164:741-747

6. Chandra D, Parisini E, Mozaffarian D (2009) Metaanalysis: travel and risk for venous thromboembolism. Ann Intern Med 151:180-190

7. Dinger J, Assmann A, Möhner S, Do Minh T (2010) Risk of venous thromboembolism and the use of dienogest- and drospirenone-containing oral contraceptives: results from a German case-control study. J Fam Plann Reprod Health Care 36(3):123-129

9. Dinger JC, Heinemann LA, Kuhl-Habich D (2007) The safety of a drospirenone-containing oral contraceptive: final results from the European active surveillance study on oral contraceptives based on 142,475 women-years of observation. Contraception 75:344-354

10. Elliott TC, Montoya CC (2008) How does VTE risk for the patch and vaginal ring compare with oral contraceptives? J Fam Pract 57:680-685

19. Gomes MPV, Deitcher SR (2004) Risk of venous thromboembolic disease associated with hormonal contraceptives and hormone replacement therapy. Arch Intern Med 164:1965-1976

21. Heinemann LAJ, Dinger JC (2007) Range of published estimates of venous thromboembolism incidence in young women. Contraception 75:328-336

22. Heit JA, Kobbervig CE, James AH et al (2005) Trends in the incidence of venous thromboembolism during pregnancy or postpartum: a 30-year population-based study. Ann Intern Med 143(10):697-706

24. James AH (2009) Venous thromboembolism in pregnancy. Arterioscler Thromb Vasc Biol 29:326-331

25. James AH, Jamison MG, Biswas MS et al (2006) Acute myocardial infarction in pregnancy: a United State population-based study. Circulation 113:1564-1571

31. Levine JS, Branch DW, Rauch J (2002) The antiphospholipid syndrome. N Engl J Med 346(10):752-763

36. Lidegaard O, Lokkegaard E, Svendsen AL et al (2009) Hormonal contraception and risk of venous thromboembolism: National follow-up study. BMJ 339:b2890-2897

38. Lowe GD (2008) Common risk factors for both arterial and venous thrombosis. $\mathrm{Br} J$ Haematol 140:488-495

40. Marik PE, Plante LA (2008) Venous thromboembolic disease and pregnancy. N Engl J Med 359:20252033

41. Martinelli I, Taioli E, Battaglioli T et al (2003) Risk of venous thromboembolism after air travel: interaction with thrombophilia and oral contraceptives. Arch Intern Med 163:2771-2774

44. Nightingale AL, Lawrenson RA, Simpson EL et al (2000) The effects of age, body mass index, smoking and general health on the risk of venous thromboembolism in users of combined oral contraceptives. Eur J Contracept Reprod Health Care 5:265-274
47. Pomp ER, Rosendaal FR, Doggen CJM (2008) Smoking increases the risk of venous thrombosis and acts synergistically with oral contraceptive use. Am J Hematol 83:97-102

48. Pomp ER, Lenselink AM, Rosendaal FR, Doggen CJM (2008) Pregnancy, the postpartum period and prothrombotic defects: risk of venous thrombosis in the MEGA study. JThromb Haemost 6:632-637

49. Pomp ER, Cessie S le, Rosendaal FR, Doggen CJR (2007) Risk of venous thrombosis: obesity and its joint effect with oral contraceptive use and prothrombotic mutations. Br J Haematol 139:289-296

50. Romero A, Alonso C, Rincon M et al (2005) Risk of venous thromboembolic disease in women. A qualitative systematic review. Eur J Obstet Gynecol Reprod Biol 121:8-17

54. Samuelsson E, Hedenmalm K, Persson I (2005) Mortality from venous thromboembolism in young Swedish women and its relation to pregnancy and use of oral contraceptives - an approach to specifying rates. Eur J Epidemiol 20(6):509-516

55. Samuelsson E, Hägg S (2004) Incidence of venous thromboembolism in young Swedish women and possibly preventable cases among combined oral contraceptive users. Acta Obstet Gynecol Scand 83:674-681

56. Seeger JD, Loughlin J, Eng PM et al (2007) Risk of thromboembolism in women taking ethinylestra$\mathrm{diol} /$ drospirenone and other oral contraceptives. Obstet Gynecol 110:587-593

57. Sehovic N, Smith KP (2010) Risk of venous thromboembolism with drospirenone in combined oral contraceptive products. Ann Pharmacother 44(5):898-903

58. Severinsen MT, Kristensen SR, Overvad K et al (2010) Venous thromboembolism discharge diagnoses in the Danish National Patient Registry should be used with caution. J Clin Epidemiol 63:223-228

63. Suissa S, Spitzer WO, Rainville B et al (2000) Recurrent use of newer oral contraceptives and the risk of venous thromboembolism. Hum Reprod 15:817-821

68. The Writing Group for the 3rd European Conference on Sex Steroids and Cardiovascular Diseases (2003). The European Consensus Development Conference 2002: Sex steroids and cardiovascular diseases. On the route to combined evidence from OC and HRT/ ERT. Maturitas 44:69-82

70. Hylckama Vlieg A van, Helmerhorst FM, Vandenbroucke JP et al (2009) The venous thrombotic risk of oral contraceptives, effects of oestrogen dose and progestogen type: results of the mega casecontrol study. BMJ 339:b2921

71. Vandenbroucke JP, Rosing J, Bloemenkamp KW et al (2001) Oral contraceptives and the risk of venous thrombosis. N Engl J Med 344:1527-1535

Interessenkonflikt. Keine Angaben. 